\title{
A teoria ambientalista no ensino e na prática profissional em enfermagem: uma revisão integrativa
}

\section{Environmental theory in teaching and professional practice in nursing: an integrative review}

\author{
${ }^{1}$ Aline Silveira-Alves alinesilveira-alves@hotmail.com \\ ${ }^{1}$ Valquiria Jorge Sepp \\ ${ }^{1}$ Lucrecia Helena Loureiro \\ ${ }^{1}$ Ilda Cecilia Moreira da Silva
}

\section{RESUMO}

A Teoria Ambientalista é uma teoria desenvolvida por Florence Nightingale em sua participação na Guerra da Crimeia em 1854. Sua teoria tinha como foco o meio ambiente relatando que todas condições do meio podem influenciar o processo de saúde. Sua teoria conceitua que o ambiente influencia diretamente a recuperação de seu paciente, então a teoria preconiza os fatores ideais para a organização do seu ambiente são eles: ventilação, iluminação, limpeza, ruídos, odores e alimentação. A revisão foi realizada por meio dos periódicos da Capes, BVS e Scielo. Para selecioná-los utilizou-se como descritores as palavras-chave enfermagem e teoria ambientalista, adotando a combinação enfermagem and teoria ambientalista. Foram localizados 57 artigos, mas só foram utilizados 5 artigos mais relevantes ao tema proposto. Ficou evidenciado que há pouquíssimos artigos relacionados ao ensino da teoria ambientalista tanto na teoria quanto na pratica.

Palavras-chave: Enfermagem. Teoria Ambientalista. Ensino.

\begin{abstract}
The Environmentalist Theory is a theory developed by Florence Nightingale in her participation in the Crimean War in 1854. Her theory focused on the environment, reporting that all conditions in the environment can influence the health process. His theory conceptualizes that the environment directly influences the recovery of his patient, so the theory advocates the ideal factors for the organization of his environment are: ventilation, lighting, cleaning, noise, odors and food. The review was carried out through the periodicals of Capes, BVS and Scielo. To select them, the key words nursing and environmental theory were used as descriptors, adopting the combination of nursing and environmental theory. We found 57 articles, but only 5 articles were found that were more relevant to the proposed themes. It was evidenced that there are very few articles related to the teaching of environmental theory in both theory and practice.
\end{abstract}

Keywords: Nursing. Environmental theory. Teaching.

1 Centro Universitário de Volta Redonda - UniFOA.. 


\section{INTRODUÇÃO}

A Teoria Ambientalista é uma teoria desenvolvida por Florence Nightingale em sua participação na Guer ra da Crimeia em 1854. Sua teoria tinha como foco o meio ambiente relatando que todas as condições do meio podem influenciar o processo de saúde (Medeiros et al, 2015). Sua teoria conceitua que o ambiente influencia diretamente a recuperação de seu paciente, então a teoria preconiza os fatores ideais para a organização do seu ambiente são eles: ventilação, iluminação, limpeza, ruídos, odores e alimentação (Martins e Benito,2016). Para Florence a enfermagem era uma arte que deveria ter treinamento prático, organizado e cientifico. A enfermeira deveria ser capacitada a servir a medicina, higiene e a cirurgia (Costa et al, 2009).

Após o seu retorno da Guerra da Criméia, por ser uma personalidade conhecida em seu país pelos seus atos e o desenvolvimento da Teoria Ambientalista, o governo inglês criou em 1860 a Escola de Enfermagem Florence no Hospital Sant Tomas em Londres (Borges et al, 2000). Essa escola surgiu devido a demanda de treinar pessoas a realizar a assistência de enfermagem e discipli nar a conduta da enfermeira. O trabalho de Florence e seus ensinamentos foram difundidos para vários países (Borges et al, 2000).

Os principais pressupostos de Nightingale para uma reflexão crítica acerca da arte de cuidar se basearam em cinco pontos fundamentais, considerados por ela como essenciais para a obtenção, por exemplo, de uma habitação saudável: “ar puro, água pura, drenagem eficiente, limpeza e luz” (Nightingale, 1992).

No entendimento de Dossey (2009), os princípios da filosofia fundamental de Nightingale são: “a cura, a liderança e a ação global”, além disso afirma que "seu princípio básico era a cura, e secundários a ele estão os princípios de liderança e da ação global, necessários para apoiar a cura em seu nível mais profundo”.

Na verdade, o trabalho de Florence influenciou a profissão e o ensino de enfermagem. Para ela a enfermagem era domínio das mulheres, mas se destacava como uma prática independente em seu próprio direito. Acreditava e defendia a atuação da enfermeira em acordo com os médicos, seguindo suas prescrições fielmente, sem contudo serem subservientes a eles. Para ela a profissão era um chamado e fundamentou o ensino na antecipação e no preenchimento das necessidades dos pacientes, atuando de forma a satisfazer tais necessidades (Dossey, 2009).

Já no Brasil a Teoria Ambientalista chegou no início do século XX, junto aos problemas sanitários que vieram juntamente ao crescimento urbano desordenado das grandes cidades (Kruse, 2006, Silveira-Alves et al 2020), quando a implantação de um modelo sanitarista se impôs diante da necessidade de se discutir a saúde como um problema nacional, sendo este o momento em que a Teoria Ambientalista chega por intermédio da Fundação Rockfeller (Kruse,2006).

O presente estudo tem como objetivo demonstrar por meio de uma revisão integrativa a influência da Teoria Ambientalista de Florence Nightingale na formação de enfermeiros e em sua prática profissional.

\section{MÉTODOS}

Este artigo se trata de uma revisão integrativa, que tem como finalidade demostrar o desenho da pesquisa, com o objetivo de aprofundar e entender sobre o fenômeno estudado (Whittemore; Knafl,2005). Esta metodologia permite que o pesquisador inclua de forma simultânea pesquisa quase-experimental e experimental, o que proporciona um maior conjunto universo de dados oriundos tanto da literatura teórica quanto empírica, o que leva a uma visão mais ampla do tema abordado (Mendes et al. 2008).

A revisão integrativa foi realizada em cinco etapas: decisão acerca dos descritores, critérios de exclusão e inclusão, avaliação dos estudos escolhidos, interpretação dos dados e síntese dos dados. 
Os dados foram retirados por buscas de artigos nas bases da Capes, Scientific Electronic Library Online (SciELO) e Biblioteca Virtual em Saúde (BVS). Para selecioná-los utilizou-se como descritores as palavras-chave enfermagem e teoria ambientalista, adotando a combinação enfermagem and teoria ambientalista.

Os artigos escolhidos atenderam aos seguintes critérios de inclusão: aderência ao assunto. serem artigos científicos completos (originais ou de revisão) e serem produzidos por pesquisadores brasileiros, para desta forma caracterizarmos o cenário nacional.

\section{RESULTADOS E DISCUSSÃO}

Foram analisados nas bases de dados pesquisadas 57 artigos. Após a leitura os títulos e resumos 52 artigos foram retirados da pesquisa por não atenderem ao critério de inclusão de forma que cinco publicações compõem o presente estudo (Fig.1)

Figura 1. Fluxograma do processo de seleção dos artigos desta revisão integrativa

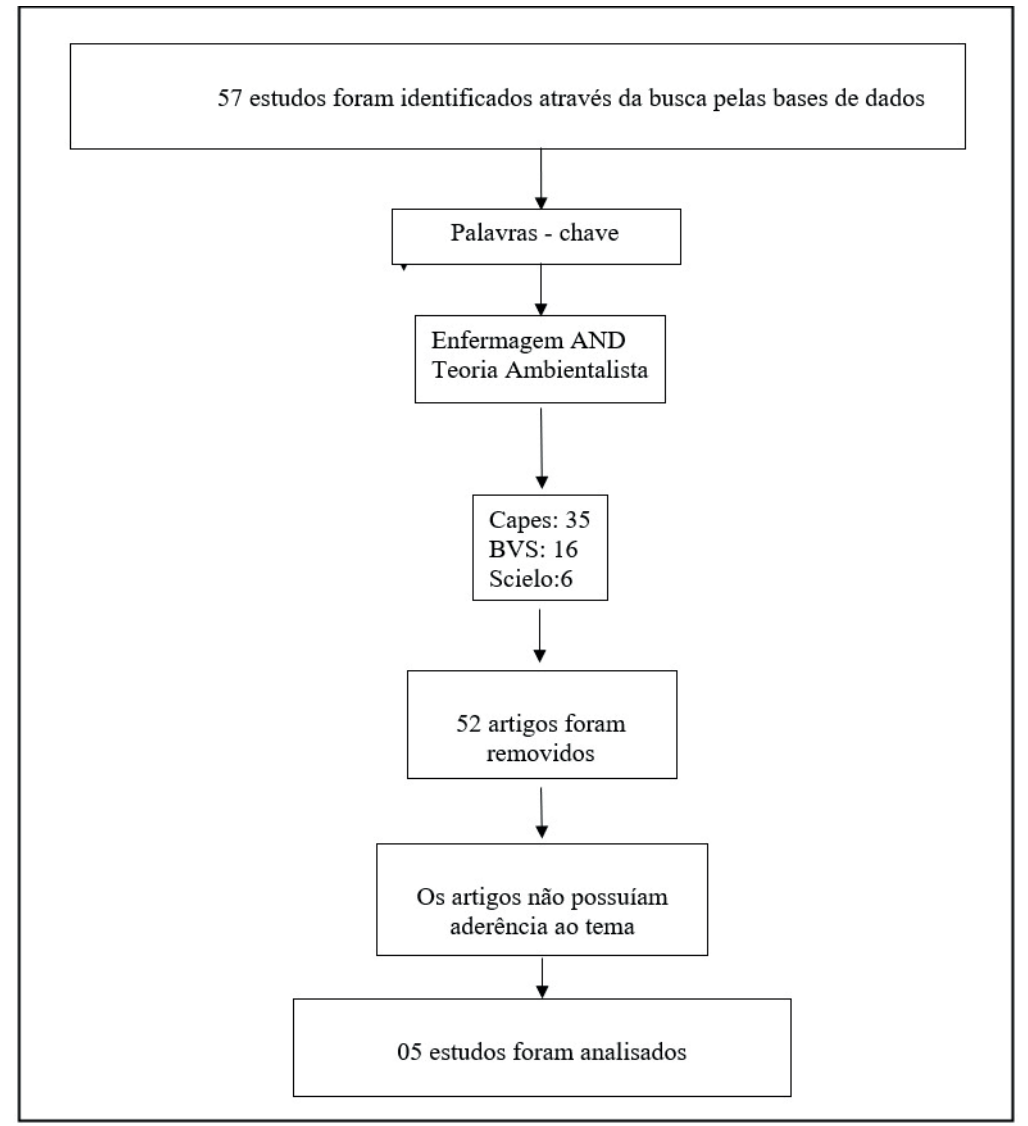

Fonte: Elaborado pela autora (2019). 
Para organizar as informações foi criado um instrumento para coletar os dados contendo: ano, periódico, título, idioma, origem e método.

Quadro1. Descrição dos estudos incluídos na revisão integrativa, ano de publicação, periódico, título, idioma, origem e método.

\begin{tabular}{|c|c|c|c|c|c|c|}
\hline & Ano & Revista/ Qualis & Nome do Artigo & Idioma & Origem & Método \\
\hline A1 & 2015 & $\begin{array}{l}\text { Escola Anna Nery Revista } \\
\text { de Enfermagem } \\
\text { Qualis: A2 (ensino) } \\
\text { B1 (enfermagem) }\end{array}$ & $\begin{array}{l}\text { Teoria Ambientalista de } \\
\text { Florence Nightingale: } \\
\text { Uma Análise Crítica }\end{array}$ & Português & $\begin{array}{l}\text { Rio de } \\
\text { Janeiro }\end{array}$ & $\begin{array}{l}\text { Teórico } \\
\text { Reflexivo }\end{array}$ \\
\hline A2 & 2011 & $\begin{array}{l}\text { Escola Anna Nery Revista } \\
\text { de Enfermagem } \\
\text { Qualis: A2 (ensino) B1 } \\
\text { (enfermagem) }\end{array}$ & $\begin{array}{l}\text { A Teoria Ambientalista } \\
\text { de Florence Nightingale } \\
\text { no Ensino da Escola de } \\
\text { Enfermagem Anna Nery } \\
(1962-1968)\end{array}$ & Português & $\begin{array}{l}\text { Rio de } \\
\text { Janeiro }\end{array}$ & $\begin{array}{l}\text { Histórico } \\
\text { Social }\end{array}$ \\
\hline A3 & 2018 & $\begin{array}{l}\text { Enfermagem Foco } \\
\text { Qualis: B2 (enfermagem) }\end{array}$ & $\begin{array}{l}\text { Análise Descritiva da } \\
\text { Teoria Ambientalista de } \\
\text { Enfermagem }\end{array}$ & Português & $\begin{array}{l}\text { Rio Grande } \\
\text { do Norte }\end{array}$ & $\begin{array}{l}\text { Teórico } \\
\text { Reflexivo }\end{array}$ \\
\hline A4 & 2016 & $\begin{array}{l}\text { Universitas Ciências da } \\
\text { Saúde } \\
\text { Qualis: B4 (enfermagem) }\end{array}$ & $\begin{array}{l}\text { Florence Nightingale e } \\
\text { suas Contribuições para } \\
\text { o controle das infecções } \\
\text { hospitalares }\end{array}$ & Português & Brasília & $\begin{array}{l}\text { Revisão } \\
\text { Sistemática }\end{array}$ \\
\hline A5 & 2013 & $\begin{array}{l}\text { Revista Gaúcha de } \\
\text { Enfermagem } \\
\text { Qualis: B1 (enfermagem) } \\
\text { B3 (ensino) }\end{array}$ & $\begin{array}{l}\text { Demandas de Cuidado } \\
\text { Domiciliar da Criança } \\
\text { Nascida Exposta ao } \\
\text { HIV na ótica da Teoria } \\
\text { Ambientalista }\end{array}$ & Português & $\begin{array}{l}\text { Rio Grande } \\
\text { do Sul }\end{array}$ & $\begin{array}{l}\text { Qualitativo } \\
\text { Exploratório } \\
\text { Descritivo }\end{array}$ \\
\hline
\end{tabular}

Fonte: Elaborado pela autora (2019).

Na presente revisão integrativa observa-se publicações entre os anos de 2011 a 2018, em português com predominância no estado do Rio de Janeiro. O levantamento bibliográfico evidenciou uma quantidade reduzida de artigos recentes sobre a teoria ambientalista no Brasil, demonstrando que existe uma clara lacuna na literatura sobre este tema.

Dentre os trabalhos selecionados, Medeiros et al. (2015) analisam a teoria ambientalista e como seu ensino influencia na pratica profissional,. demonstrando que a teoria ambientalista tem conceitos ainda extremamente atuais e como tal sendo de importância significativa para a atual prática do enfermeiro. Os autores ainda apontam que os conceitos da teoria fornecem uma base conceitual sólida para a elaboração de proposições para uma prática de higiene ambiental pelos profissionais da enfermagem.

O trabalho de Haddad et al. (2015), por sua vez, já se debruça na discussão sobre a aplicação dos conceitos da teoria ambientalista na disciplina de fundamentos de enfermagem. Os autores demonstram em seu estudo que a teoria colabora em evidenciar a importância da implementação de assistência por condições de recuperação, reabilitação e cura através de um ambiente de qualidade, além da importância da pratica de um cuidado holístico através de uma assistência sistemática dos princípios éticos da profissão e o valores morais dessa sociedade. Os 
autores ainda ressaltam que diversos aspectos da teoria ambientalista foram incorporados ao ensino da prática de enfermagem em diferentes partes do mundo, inclusive no Brasil, sendo os ideais de Florence Nightingale adaptados às diferentes realidades nacionais, cabendo aos prof ssionais de enfermagem o papel de analisar as necessidades de suas sociedades e assim praticarem a prof ssão da melhor forma dentro de seu contexto de época.

Bezerra et al. (2018) discutem em seu trabalho a avaliação da teoria ambientalista sob o prisma da teoria de Meleis, que permite explicitar os principais componentes da estrutura de uma teoria. A análise da teoria ambientalista sob a perspectiva de Meleis mostrou a importância da ação do prof ssional da enfermagem implementado um ambiente saudável. Os autores concluem que a teoria se mostra sempre atual, mostrando sua aplicabilidade no dia-a-dia na pratica prof ssional relacionando o ser humano, a saúde e o ambiente.

Martins \& Benito (2016) discutem em seu artigo como a teoria ambientalista contribui no controle e combate de infecções hospitalares, através da arquitetura hospitalar e sua administração, demonstrando a importância da teoria ambientalista através da atenção direcionada aos pacientem em todas as ações hospitalares.

Por $\mathrm{fm}$, Lima et al. (2013) discutem sobre a necessidade de identif car as demandas dos cuidados domiciliares da criança exposta a doença do HIV, através da teoria ambientalista. Os autores demonstram, através da teoria, que para promover a saúde a essa criança é necessário focar no seu ambiente domiciliar, o qual deve apresentar ar puro, água fltrada, rede de esgoto e saneamento básico bem como iluminação e ventilação adequadas, características essas que colaboram para a melhoria da saúde destas crianças.

\section{CONSIDERAÇÕES FINAIS}

A teoria ambientalista de Florence Nightingale representa ainda hoje um marco teórico na enfermagem e mais de um século após a sua proposição ela ainda se mostra atual, de forma que a importância de seu ensino e presença na prática da enfermagem norteada por higiene ambiental. Os estudos abordados nesta revisão integrativa abordam principalmente a estrutura da teoria e suas aplicações, sendo um único trabalho dedicado ao ensino da teoria, algo que evidencia que apesar de sua importância a forma que esta é ensinada aos graduandos é pouco abordada na literatura.

\section{REFERÊNCIA}

Bezerra, C. et al. Análise descritiva da teoria ambientalista de enfermagem. Enfermagem em Foco, v. 9, n.2,p. $79-83,2018$.

Borges, E. et al. Ref exões sobre enfermagem pós-Florence - Revista Mineira de Enfermagem, v. 4, n. 2, p. $77-82,2000$.

Costa, R. et.al. O legado de Florence Nightingale: uma viagem no tempo. Texto \& Contexto - Enfermagem, v. 18, n. 4, p. 661-69, 2019.

Dossey, B.M. Ciência dos seres humanos unitários e irredutíveis In McEwen, M. Bases teóricas de enfermagem, McEwen, M.; Wills $2^{\mathrm{a}}$ ed., Porto Alegre, Artmed, 2009.

Haddad, V.C.N. A teoria ambientalista de Florence Nightingale no ensino da escola de enfermagem anna nery (1962 - 1968). Esc. Anna Nery Rev. Enferm, v. 15, n. 4, p. 755-761, 2011. 
Kruse, M.H. Enfermagem Moderna: A ordem do cuidado. Revista Brasileira de Enfermagem, 59, p. 403-410, 2006.

Lima,I.C. et al. Demandas de cuidado domiciliar da criança nascida exposta ao HIV na ótica da teoria ambientalista. Rev. Gaúcha Enferm., 2013; 34(3): 64-71.

Martins, D. F., Lincoln A. O. B. Florence Nightingale e as suas contribuições para o controle das infecções hospitalares. Universitas: Ciências da Saúde, 14, n. 2, 2016.

Medeiros, A., Eders, B. e Lira, A. Teoria Ambientalista de Florence Nightingale: Uma Análise Critica. Escola Anna Nery de Enfermagem, 19, n. 3, p. 518-21, 2015.

Mendes K.D,S, Silveira R.C.C.P, Galvão C.M. Revisão integrativa: método de pesquisa para a incorporação de evidências na saúde e na enfermagem. Texto Contexto Enfermagem,; 17(4):758-64, 2008.

Nightingale, F. Notes on nursing: what it is and what it is not. Lippincott Williams \& Wilkins, 1992.

Silveira-Alves, A.; Figueiró, R.; Loureiro, L.H.; Silva, I.C.M. A história do cuidado desde suas origens até os tempos de pandemia. Acta Biomedica Brasiliensia. v. 11, n. 1, p. 5 - 8, 2020. 